\title{
Injury-elicited stressors alter endogenous retrovirus expression in lymphocytes depending on cell type and source lymphoid organ
}

\author{
Kang-Hoon Lee, Debora Lim, Tajia Green, David Greenhalgh and Kiho Cho*
}

\begin{abstract}
Background: Murine leukemia virus-type endogenous retroviruses (MuLV-ERVs) constitute $~ 10 \%$ of the mouse genome and are associated with various pathophysiologic processes. In this study, we examined whether MuLV-ERVs' response to burn-elicited stressors is specific for certain lymphocyte populations and/or locations of lymphoid organ.

Results: B- and T-cells, which were sorted from nine lymphoid organs of C57BL/6J mice after burn, were subjected to MuLV-ERV expression analyses. Overall, the post-burn MuLV-ERV expression pattern was dependent on lymphocyte type, time after injury, location of lymphoid organ, and MuLV-ERV type. For instance, the MuLV-ERV expression in T-cells from the thymus and three cervical lymph nodes decreased at 3 hours post-burn while the expression of some MuLV-ERVs was augmented in B-cells derived from the mesenteric lymph node. The MuLV-ERV U3 sequences population of the burn-24 hours group was less diverse in comparison to the no burn and burn-3 hours groups. In addition, it was apparent that at the 24 hours time point, the U3 populations of B-cells from both no burn and burn groups were less heterogeneous than the T-cells' U3 populations. Using the U3 sequences, some of which were isolated only from specific experimental groups (B- vs. T-cells; no burn vs. burn), as probes, 51 putative MuLV-ERVs, including 16 full-length proviruses, were mapped followed by characterization of their biologic properties.
\end{abstract}

Conclusion: MuLV-ERVs' response to burn-elicited stressors may be differentially controlled depending on lymphocyte type, location of lymphoid organ, MuLV-ERV type, and stress duration.

Keywords: B-cell, T-cell, Burn, Stress, Murine leukemia virus-type endogenous retrovirus

\section{Background}

Proviral sequences of the murine leukemia virus-type endogenous retroviruses (MuLV-ERVs) are genomic footprints reflecting the ancient infection of germ-line cells and are presumed to constitute $\sim 10 \%$ of the mouse genome [1]. Although most MuLV-ERV genomes are reported to be defective primarily due to mutation and recombination events, some retain intact proviral genome features, such as identical long terminal repeats (LTRs) at both ends, which are formed by retroviruses' unique replication process, and coding potential for the major structural genes (gag, pol and env) which are

\footnotetext{
* Correspondence: kcho@ucdavis.edu

Department of Surgery, Burn Research, Shriners Hospitals for Children Northern California, University of California, Davis, Sacramento, CA 95817, USA
}

\section{Biomed Central}

essential for virion assembly [2-4]. Expression of MuLVERVs is mostly controlled by their U3 promoters located on the $5^{\prime}$ LTR, in conjunction with the transcription machinery that is dynamically and uniquely formed within individual cells [5].

Recent studies provide evidence that human endogenous retroviruses (HERVs) are involved in normal physiology as well as various disease processes [6]. The HERV-W envelope protein, called syncytin, is highly expressed in the syncytiotrophoblast layer and plays a critical role in placental differentiation [7]. In addition, the inflammatory properties of syncytin contribute to the degeneration of oligodendrocytes responsible for the development of multiple sclerosis, an autoimmune disease [8]. 
Patients with severe burn injury often succumb to systemic immune disorder in conjunction with internal organ damage [9]. However, the mechanisms underlying the post-burn pathologic changes have not yet been fully characterized. The results from recent studies suggest that post-burn systemic inflammatory response is associated with the activation and/or depletion of specific lymphocyte subpopulations, such as $\mathrm{CD} 4{ }^{+} \mathrm{CD} 25^{+} \mathrm{T}$ regulatory cells, lymphocytes expressing CD25, CD69 and CD71, NK-T-cells, and CD8 ${ }^{+}$T-cells [10-12]. Sepsiselicited stressors induce apoptosis in the spleen, resulting in the depletion of B-cells and accompanying immune malfunction [13]. However, the central signaling molecules responsible for the post-burn activation and/or depletion of lymphocytes remain to be elucidated.

Our recent studies demonstrated that burn-elicited stressors differentially alter the expression of MuLV-ERVs in a range of internal organs, including various lymphoid tissues, in a ERV type- and organ-specific manner [14]. Moreover, some MuLV-ERV envelope proteins were able to modulate the mRNA expression of inflammatory cytokines such as IL- 6 and IL-1 $\beta$ in macrophage cells [15]. In this study, we hypothesize that the differential post-burn alterations in MuLV-ERV expression, depending on lymphocyte types and/or lymphoid organs, may be associated with systemic pathogeneses, including immune disorder. We examined whether MuLV-ERVs' response to burn-elicited stressors is specific depending on lymphocyte type and the location of lymphoid organ followed by an investigation of the biologic characteristics of the relevant MuLV-ERVs.

\section{Methods}

\section{Animal experiments}

The experimental protocols involving mice were approved by the Animal Use and Care Administrative Advisory Committee of the University of California, Davis. Female C57BL/6J mice (12 weeks old) were purchased from Jackson Laboratory-West (Sacramento, CA). The baseline MuLV-ERV expression patterns were examined in nine lymphoid organs (spleen, thymus, and seven lymph nodes [axillary, inguinal, mesenteric and pooled cervicals \{mandibular, accessory mandibular, superficial parotid, and deep cervical\}]). Three mice were sacrificed for tissue collection by cervical dislocation without any treatment. The burn protocol has been described previously [16]. Briefly, under general anesthesia, an $\sim 18 \%$ total body surface area flame burn was generated on the shaved back of mice followed by resuscitation with saline and buprenorphine. Control (no burn) mice were treated the same as burn mice but without the flame burn. The three mice from each experimental group were sacrificed by $\mathrm{CO}_{2}$ inhalation at 3 hours and 24 hours after burn followed by the collection of the same nine lymphoid organs as above without any pooling. The burn experiment was repeated four times.

\section{Cell sorting}

The collected tissues were minced, suspended in a trypsin/EDTA solution, and then passed through a cell strainer (BD Biosciences, CA). The cells were washed with PBS containing $0.1 \%$ BSA followed by centrifugation at $1,000 \times g$ for $5 \mathrm{~min}$ at $4^{\circ} \mathrm{C}$. Following the removal of red blood cells, cells were sorted into B- and T-cells using pan-B and pan-T Dynabeads (Invitrogen, Carlsbad, $\mathrm{CA}$ ), respectively, according to the protocols provided by the manufacturer.

\section{Total RNA isolation and RT-PCR}

Total RNAs were isolated from the sorted cells using an RNeasy Mini Kit (Qiagen, Valencia, CA) following homogenization using a QIAshredder (Qiagen). cDNAs were synthesized using Sensiscript reverse transcriptase (Qiagen), RNase inhibitor (Promega, Madison, WI) and an oligo-dT primer (5'-GGC CAC GCG TCG ACT AGT ACT TTT TTT TTT TTT TTT T- 3'). A set of primers, ERV-U1 (5'-CGG GCG ACT CAG TCT ATC GG-3') and ERV-U2 (5'-CAG TAT CAC CAA CTC AAA TC-3'), was used to amplify the MuLV-ERV U3 regions [17]. $\beta$-actin, serving as a normalization control, was amplified with the primer set: $5^{\prime}$-CCA ACT GGG ACG ACA TGG AA-3' and 5'-GTA GAT GGG CAC AGT GTG GG-3'.

\section{Measurement of relative purity of sorted cell populations}

The relative purity of the sorted cell populations was measured by real-time RT-PCR using CD20 (B-cell antigen) primers (5'-AAA ACC TCC AGG AAG AGT TTG GTC-3' and 5'-CGA TCT CAT TTT CCA CTG GCA AG-3') for B-cells and Thy1 (Thymus cell antigen-1) primers (5'-TCC TGC TCT CAG TCT TGC-3' and $5^{\prime}$ AGT AGT CGC CCT CAT CCT TG-3') for T-cells. Real-time RT-PCR was performed using the Brilliant-III SYBR Green QPCR kit from Agilent Technologies (La Jolla, CA) and the results were analyzed using the MxPro (v.4.10) program (Agilent Technologies). Cell type specific mRNA levels (CD20 and Thy1) were calculated from each sorted cell population with the level of $\beta$-actin as a normalization control, using the same primer set as above. The relative purity of each cell population was calculated with the assumption that there are only B- and T-cells in each sorted cell population and CD20 and Thy1 are exclusively expressed in B-cells and T-cells, respectively.

\section{Densitometry and statistical analysis}

The density of the PCR products was measured using KODAK Molecular Imaging Software Version 4.5.1 
(Eastman Kodak Company, Rochester, NY). All optical densities were measured based on mass values with the Hi-Lo ${ }^{\text {TM }}$ DNA marker (a gift from Minnesota Molecular Inc., Minneapolis, MN). The density of each MuLV-ERV U3 band was normalized to its corresponding $\beta$-actin value to obtain the relative intensity of each U3 band. Differences in the relative intensity of the matching MuLV-ERV U3 bands between no burn and burn were calculated as fold changes. Statistical significance $(\mathrm{p}<$ 0.05 ) of fold changes between the matching pairs (no burn and burn) of the individual U3 bands was determined using the data from four burn experiments and Student's $t$-test.

\section{Cloning and sequencing}

Eighty-eight MuLV-ERV U3 PCR amplicons/bands, which had contrasting densities between individual no burn and burn pairs, were selected for sequence analyses. The PCR amplicons were purified using the QIAquick Gel Extraction kit (Qiagen) and then cloned into the pGEM $^{\circledR}$-T Easy vector (Promega). Three clones were prepared from each U3 PCR amplicon/band. The plasmid DNAs were prepared using a QIAprep Spin Miniprep kit (Qiagen) for sequencing analysis. Sequencing was performed at a core sequencing facility at the University of California, Davis.

\section{Alignment and phylogenetic analyses}

From a total of 260 U3 sequences, 100 unique U3 sequences were identified using Seqman Pro Version 8.1.2 (DNASTAR, Madison, WI). Multiple sequence alignment of the 100 unique U3s was performed using the Clustal W method in MegAlign (DNASTAR), and a phylogenetic tree was generated by the HYPERTREE program [18]. Seven U3 sequences, representing the seven phylogenetic branches, were subjected to a multiple alignment analysis to examine the U3 sequence features of direct repeats (1/ $1^{*}, 4 / 4^{*}, 5 / 5^{*}$, and $\left.6 / 6^{*}\right)$, insertion of 190 nucleotides, and unique region (2) based on the protocol by Tomonaga et al. $[17,19]$.

\section{Profiling of transcription regulatory elements}

The 100 unique MuLV-ERV U3 sequences were surveyed for putative transcription regulatory elements using the MatInspector program (Genomatix, Munich, Germany) within the vertebrate matrix group with a core similarity higher than 0.95 [20].

\section{Determination of population diversity of U3 sequences within each experimental group}

Using the MEGA4 program [21], the population diversity of U3 sequences was measured in each experimental group and compared among different groups in two analyses: a) no burn, burn-3 hours, and burn-24 hours, and b) no burn-B-cells, burn-24 hours-B-cells, no burn-Tcells, and burn-24 hours-T-cells.

\section{In silico mapping of putative MuLV-ERVs and characterization of their biological properties}

The C57BL/6J mouse genome database from the $\mathrm{Na}$ tional Center for Biotechnology Information (NCBI) was surveyed for putative MuLV-ERVs with a greater than 98\% identity using the 100 unique U3 sequences as mining probes and the Advanced BLAST program (NCBI). The coding potential of each MuLV-ERV was determined using the Vector NTI program (Invitrogen). Three polypeptide genes ( $g a g$, pol, and env) were noted as intact $(+)$, partial $(\mathrm{P})$, or defective $(-)$ relative to the references (AY219567.2 and AF033811). Each MuLVERV was mapped with the chromosomal location and strand orientation. The primer binding site (PBS) of each MuLV-ERV was identified by examination of a stretch of 18 nucleotides immediately downstream of the $5^{\prime}$ LTR region [22]. In addition, the annotated gene(s) within $100 \mathrm{~Kb}$ upstream and downstream of each MuLV-ERV locus, if any, were mapped.

\section{Results and discussion}

Lymphocyte type-specific MuLV-ERV expression patterns in lymphoid organs of normal mice

Prior to the investigation of post-burn alterations in MuLV-ERV expression in B- and T-cells of different lymphoid organs (spleen, thymus, axillary lymph node $[\mathrm{LN}]$, inguinal LN, mesenteric LN, and cervical LNs), baseline MuLV-ERV expression levels were examined in B- and T-cells isolated from $\sim 12$ week old female C57BL/6J mice. The relative purity of the sorted cell populations was determined by measuring CD20 (for Bcells) and Thy1 (for T-cells) levels by real-time RT-PCR. The relative purity of the sorted B- and T-cell populations was greater than 93\%, except for the B-cell population in the thymus ( $65 \%)$. Throughout the lymphoid organs examined, the MuLV-ERV expression pattern, which was examined by RT-PCR amplification of the $3^{\prime}$ U3 regions, was substantially different between B- and T-cells (Figure 1). The expression pattern of an intense $\sim 500 \mathrm{bp}$ and two $(\sim 750 \mathrm{bp}$ and $\sim 450 \mathrm{bp})$ lighter U3 bands was shared by B-cells derived from all lymphoid organs examined. In contrast, four distinct U3 bands, ranging from $\sim 500 \mathrm{bp}$ to $\sim 750 \mathrm{bp}$, were amplified in $\mathrm{T}$ cells with variable intensity depending on the source lymphoid organs. It was interesting to note that the $\sim 500$ bp U3 band was more intense in B-cells from all four LN samples compared to B-cells from the spleen and thymus. The findings from this study suggest that the expression of MuLV-ERVs is differentially regulated depending on the lymphocyte type, source lymphoid organ, and ERV type. 


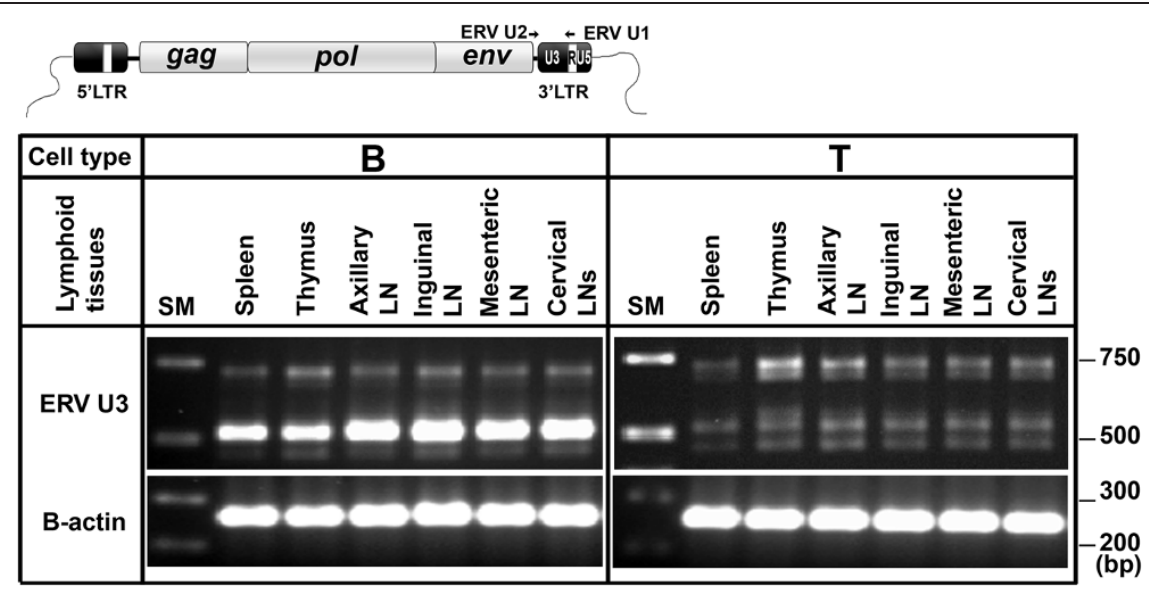

Figure 1 MuLV-ERV expression in B- and T-cells derived from lymphoid organs of C57BL/6J mice. MuLV-ERV expression was examined in B- and T-cells isolated from the spleen, thymus, and seven different lymph nodes of female C57BL/6J mice without any treatment. A schematic drawing of a typical MuLV-ERV proviral structure indicates the relative locations of a set of primers used for amplification of the U3 regions. Differential MuLV-ERV expression patterns were observed in B- and T-cells derived from various lymphoid organs. LN (lymph node), SM (size marker).

Burn-elicited alterations in MuLV-ERV expression in lymphocytes depending on lymphocyte type, source lymphoid organ, and ERV type

The effects of burn-elicited stressors on the expression of MuLV-ERVs in the B- and T-cells from the spleen, thymus, and seven lymph nodes were examined at 3 hours and 24 hours after injury by amplifying the $3^{\prime} \mathrm{U} 3$ regions. At 3 hours post-burn, the MuLV-ERV expression, represented by four distinct U3 bands, was decreased in the T-cells from the thymus and three cervical LNs (mandibular, accessory mandibular, and superficial parotid), but not in the T-cells from the deep cervical LN (Figure 2). In contrast, there was an increase in intensity of one to two U3 bands in the Tcells from the thymus and superficial parotid cervical LN at 24 hours post-burn when there was a decrease in U3 bands in the T-cells of the spleen and mesenteric LN.

In B-cells, there was an induction of two ( $a$ and $b$ bands) of the three $\mathrm{U} 3$ bands in the mesenteric $\mathrm{LN}$ at
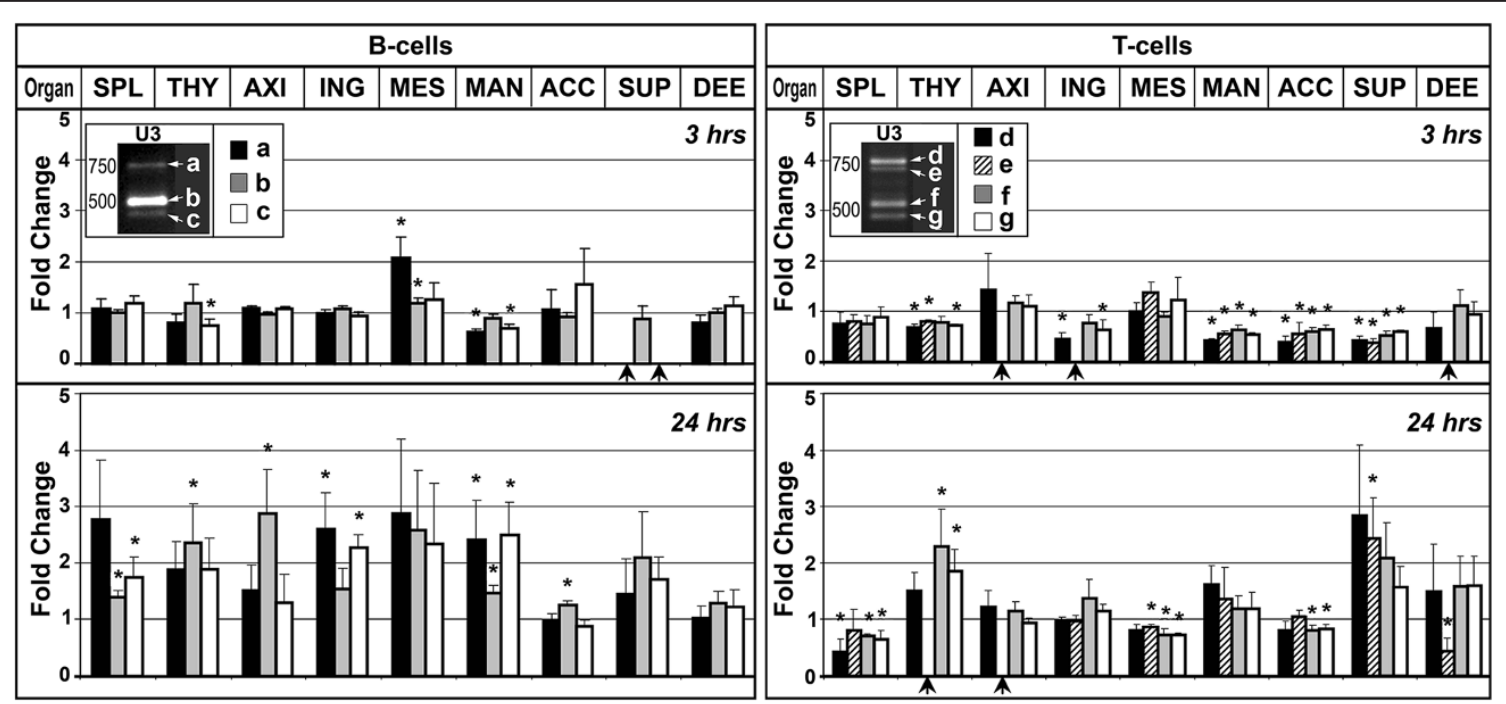

Figure 2 Post-burn alterations in the expression of MuLV-ERVs in B- and T-cells isolated from lymphoid organs of C57BL/6J mice. POstburn (3 hours and 24 hours) changes in the expression of MuLV-ERVs were measured in the B-and T-cells isolated from nine different lymphoid organs. The results, which were presented as a fold change (burn/no burn) for the individual MuLV-ERV U3 bands ( $a$, b, and c for B-cells; d, e, f, and $\mathrm{g}$ for T-cells), revealed a unique pattern for each experimental group (cell/organ/time). SPL (spleen), THY (thymus), LN (lymph node), AXI (axillary LN), ING (inguinal LN), MES (mesenteric LN), MAN (mandibular cervical LN), ACC (accessory mandibular cervical LN), SUP (superficial parotid cervical LN), DEE (deep cervical LN). A black arrow indicates unmeasured samples due to the low expression level. Error bars indicate standard error of the mean. ${ }^{*} P<0.05$. 
3 hours post-burn while two bands (a and c) were down regulated in the B-cells of the mandibular cervical LN. However, at 24 hours post-burn, the expression levels of all three U3 bands were increased in the B-cells from the mandibular cervical LN. Also at the 24 hours time point, post-burn increases in one or two U3 bands were also observed in the B-cells of the spleen, thymus, axillary $\mathrm{LN}$, inguinal $\mathrm{LN}$, and/or accessory mandibular cervical LN.

The results from this study suggest that the lymphocyte type (B- or T-cell), source lymphoid organ, and duration after injury play a role in post-burn changes (induction or repression) in the expression of MuLV-ERVs. It is possible that the post-burn differential expression of MuLV-ERVs resulted from changes in the composition of the B- and T-cell sub-populations (e.g. naïve vs. effector cells), which have different transcription environments, instead of (and/or in addition to) the affected MuLV-ERVs' altered transcriptional potentials. It was interesting to note that the post-burn increase in the MuLV-ERV expression in the B-cells from the mesenteric LN parallels the proliferation of specific B-cell populations in the mesenteric LN and spleen after burn [23].

\section{MuLV-ERV U3 sequences identified in specific lymphocyte type and/or injury (no burn/burn) condition}

Three clones from each of the $88 \mathrm{MuLV}$-ERV U3 amplicons/bands, which showed contrasting expression levels between the individual no burn and burn pairs, were sequenced to identify lymphocyte type- and/or injury (no burn/burn)-specific U3 sequences. From a total of 260 MuLV-ERV U3 sequences cloned from the entire set of MuLV-ERV U3 bands examined, 100 unique U3 sequences, ranging from 346 nucleotides to 615 nucleotides in size, were identified (Additional file 1: Table S1). An initial phylogenetic analysis resulted in seven distinct branches of U3 sequences, primarily segregated by their size (group I [largest U3s] group VII [smallest U3s]) (Figure 3). Group III (440 bp length) had the most number of U3 sequences (40) followed by group VII (346 bp length) (25 sequences) then group I (greater than $603 \mathrm{bp}$ length) (19 sequences). There were only two U3 sequences in groups IV and VI (433 bp and 361 bp in size, respectively). The group II U3 sequences (5) were isolated only from T-cells and the group VI U3 sequences (2) were unique for B-cells. On the other hand, the group IV U3 sequences (2) were identified only from no burn control mice. The other four branches (groups I, III, V, and VII) consisted of U3 sequences isolated from both lymphocyte types derived from both no burn and burn mice.

The results obtained from this study suggest that some MuLV-ERVs are selectively expressed depending on lymphocyte type (B- or T-cell) and/or presence of burnelicited stressors. However, it is possible that an expanded

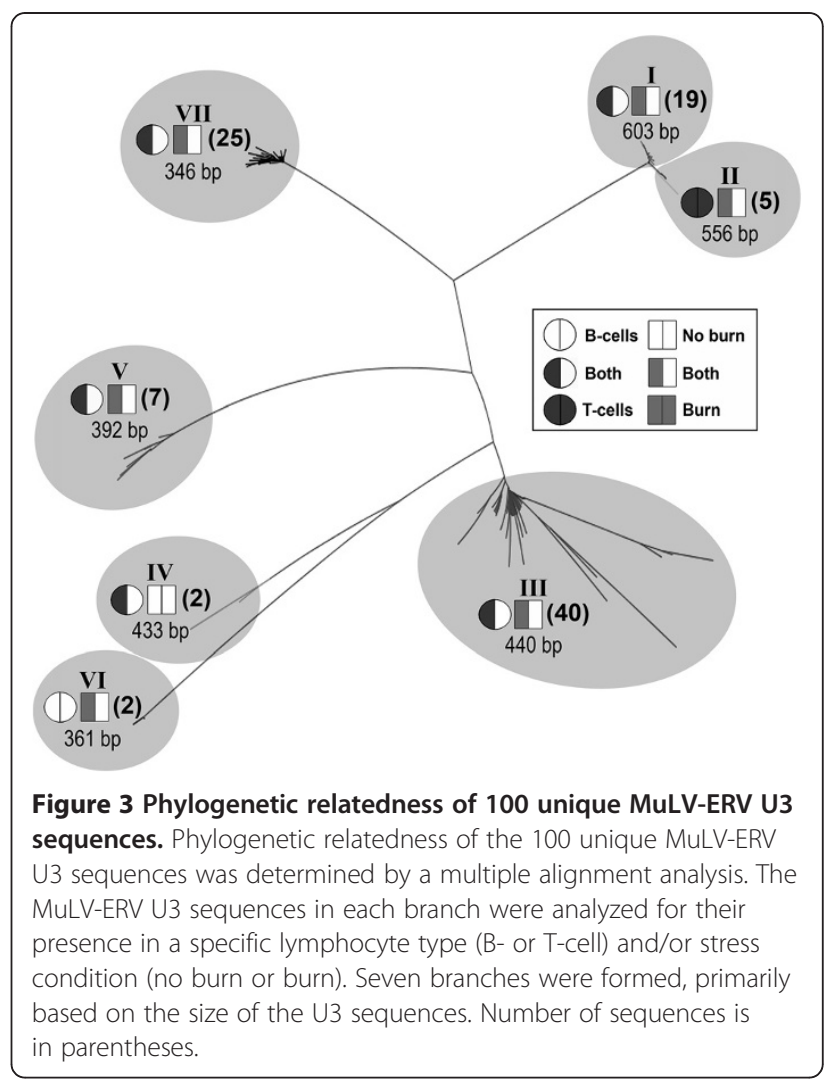

saturation cloning protocol would find that some cell type and/or burn-specific U3 sequences are not exclusively present in one group over the other. In this case, a statistical evaluation may be needed to determine the specificity of the U3 sequences in regard to the lymphocyte type and/or burn-elicited stressors.

\section{Characteristics of seven U3 sequences representing the phylogenetic branches}

Seven representative U3 sequences, which were chosen from the seven main phylogenetic branches, were subjected to an alignment analysis using reference sequences to characterize their sequence features, such as direct repeats, unique sequence, and an insertion of 190 nucleotides. These U3 sequence features were utilized as key parameters for the in silico determination of the MuLV-ERV tropism traits [19]. The U3 sequences of the groups I and II had an insertion of 190 nucleotides, which is consistent with their relatively large size compared to the other five branches (Table 1). There was a well-conserved TATA box in all seven U3 sequences; however, each U3 sequence retained a unique profile in regard to the direct repeats $\left(1 / 1^{*}, 4 / 4^{*}\right.$, $5 / 5^{*}$, and $6 / 6^{*}$ ) and the unique sequence (2). The specific features of the individual U3 sequences were translated into the tropism traits of the respective putative MuLV-ERVs $[17,19]$. However, the sequences features of the group VI U3 sequences were too diverse to predict its tropism trait. 
Table 1 Sequence characteristics and putative tropism traits of seven MuLV-ERV U3 sequences representing the seven main phylogenetic branches

\begin{tabular}{|c|c|c|c|c|c|c|c|c|c|c|}
\hline \multirow{2}{*}{ Group } & \multirow{2}{*}{ U3 Probe } & \multirow{2}{*}{ Size (bp) } & \multicolumn{5}{|c|}{ Repeat/unique region } & \multirow{2}{*}{ TATA } & \multirow{2}{*}{$\begin{array}{l}\text { Insertion } \\
\text { (190 bp) }\end{array}$} & \multirow{2}{*}{ Tropism } \\
\hline & & & $1 / 1^{*}$ & 2 & $4 / 4^{*}$ & $5 / 5^{*}$ & $6 / 6^{*}$ & & & \\
\hline 1 & $\mathrm{~B}_{3 \mathrm{H}^{-}} \mathrm{T}_{\mathrm{TH}^{-1}}$ & 603 & $+/+$ & + & + & + & $+/+$ & + & + & $P-\|$ \\
\hline III & $\mathrm{B}_{3 \mathrm{H}^{-}} \mathrm{T}_{\mathrm{NN}^{-}}-3$ & 556 & $+/+$ & $\cdot$ & + & + & $+/+$ & + & + & $P-1$ \\
\hline III & $\mathrm{B}_{3 \mathrm{H}^{-}} \mathrm{T}_{\mathrm{TH}^{-}}-7$ & 440 & $+/+$ & + & + & $+/+$ & $+/+$ & + & $\cdot$ & $X-11$ \\
\hline IV & $\mathrm{NB}_{24 \mathrm{H}^{-}} \mathrm{T}_{\mathrm{SC}}{ }^{-3}$ & 433 & $+/+$ & $\cdot$ & $+/+$ & $+/+$ & + & + & - & $x-11$ \\
\hline $\mathrm{V}$ & $\mathrm{B}_{3 \mathrm{H}^{-}} \mathrm{T}_{\mathrm{TH}^{-}}-6$ & 392 & $+/+$ & + & + & $+/+$ & $+/+$ & + & . & $x-1$ \\
\hline$\overline{\mathrm{VI}}$ & $\mathrm{B}_{3 \mathrm{H}^{-}}-\mathrm{B}_{\mathrm{ME}}-9$ & 361 & $+/+$ & $\cdot$ & . & $+/+$ & $+/+$ & + & . & . \\
\hline$\overline{\mathrm{VII}}$ & $\mathrm{B}_{3 \mathrm{H}^{-}} \mathrm{T}_{\mathrm{TH}^{-}} 11$ & 346 & $+/+$ & + & $+/+$ & . & $+/+$ & + & . & $X-11$ \\
\hline
\end{tabular}

Sequence characteristics: direct repeats $\left(1 / 1^{*}, 4 / 4^{*}, 5 / 5^{*}\right.$, and $\left.6 / 6^{*}\right)$, unique region (2), insertion of 190 nucleotides, and TATA box; $+/+($ direct repeat), $+($ single copy), $•$ (absence).

Evidence for post-burn increase in the expression of specific MuLV-ERVs

The population diversity of the U3 sequences (a total of 260) was measured in each experimental group and compared among the groups in two different analyses [21]: i) no burn, burn-3 hours, and burn-24 hours, and ii) no burn-B-cells, burn-24 hours-B-cells, no burn-Tcells, and burn-24 hours-T-cells. The results from the first diversity analysis revealed that the U3 population of the burn-24 hours group was substantially less heterogeneous compared to the other two groups (no burn and burn-3 hours) (Figure 4). In contrast, it appears that there was an increase in diversity among the U3 population in the burn-3 hours group. The finding of decreased U3 population diversity within the burn-24 hours group

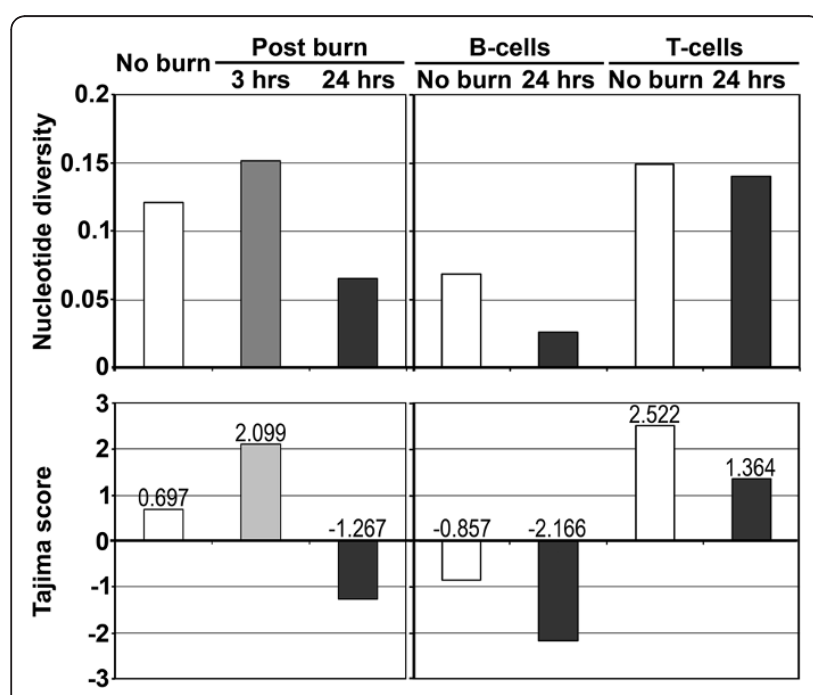

Figure 4 Decreased population diversity of MuLV-ERV U3 sequences at $\mathbf{2 4}$ hours after burn. The U3 population diversity within each experimental group was measured [21] and the relevant values, indicating different diversity levels, were presented as nucleotide diversity and Tajima statistics scores. There was a marked decrease in the U3 population diversity at 24 hours after burn compared to no burn.
Table 2 Differential transcription potentials of MuLV-ERV U3-promoter sequences

\begin{tabular}{|c|c|c|c|c|}
\hline \multirow{3}{*}{$\begin{array}{c}\text { TRE } \\
\text { DMRT }\end{array}$} & \multirow{3}{*}{$\begin{array}{c}\text { Consensus motif } \\
\text { ctttcttGTAActtttgagaa }\end{array}$} & \multirow{2}{*}{\multicolumn{3}{|c|}{$\begin{array}{c}\text { Occurrence } \\
\text { Burn/No burn }\end{array}$}} \\
\hline & & & & \\
\hline & & 68 & 1 & 1 \\
\hline BRNF & tctggatctatTGATttga & 35 & 1 & 4 \\
\hline BCL6 & ctctttcTGGAaactga & 31 & 1 & 3 \\
\hline TF2D & gctctggtactttttcatcccttgcaaaatGGCGttact & 27 & 1 & 1 \\
\hline PTF1 & gccaTCTGttcttggecctga & 28 & I & 1 \\
\hline MYT1 & tgagAGCTcagct & 27 & I & 2 \\
\hline AIRE & gtatttctcggtcatttGGGGaaactg & 19 & 1 & 0 \\
\hline ZNFP & cacaCACCtggtc & 22 & I & 0 \\
\hline NRF1 & gggGCCCaggcgcttga & 21 & I & 1 \\
\hline \multirow{2}{*}{ TRE } & \multirow{2}{*}{ Consensus motif } & \multicolumn{3}{|c|}{ Occurrence } \\
\hline & & \multicolumn{3}{|c|}{ B-cell/T-cell } \\
\hline PAX2 & gttattcggggaacctgAAACtg & 32 & 1 & 13 \\
\hline HAML & ctgaGTGGttagttc & 35 & 1 & 8 \\
\hline NKXH & acttttgaGAACttagctc & 33 & 1 & 10 \\
\hline HOMF & taatctTTAActgttaatc & 16 & 1 & 37 \\
\hline SORY & gttctaaaCAATgtctattttca & 14 & 1 & 28 \\
\hline HEAT & gagagggtctaatGAAGgttcaaca & 10 & 1 & 30 \\
\hline MAZF & atggGAGGggtac/gtacGAGGagagg & 8 & 1 & 18 \\
\hline MEF2 & acaatgtCTATtttcaagaaatg & 5 & 1 & 19 \\
\hline GATA & tttAGATctaaac & 6 & 1 & 16 \\
\hline CART & gggtcTAATgaaggttcaaca & 8 & 1 & 16 \\
\hline HOXF & gggtcTAATgaaggttcaa & 7 & 1 & 17 \\
\hline LHXF & gagagggtcTAATgaaggttcaa & 7 & I & 16 \\
\hline FXRE & AGGTgcttgacca & 5 & I & 19 \\
\hline GRHL & cacacaGGTTcaa & 7 & 1 & 16 \\
\hline NKX6 & atctTTAActgttaa & 6 & 1 & 16 \\
\hline CEBP & aaggttcaGCAAtgg & 6 & 1 & 15 \\
\hline FKHD & tagttcTAAAcaatgtc & 5 & 1 & 16 \\
\hline PDX1 & gaaagagtcTAATgaaggt & 5 & 1 & 15 \\
\hline RXRF & cacagGTTCaaggagtggccagagc & 5 & 1 & 16 \\
\hline
\end{tabular}

Numbers indicate the occurrence frequency of specific TREs (transcription regulatory elements) within the 100 unique U3-promoter sequences. Please refer to (Additional file 3: Table S3) for the complete list of TRE abbreviations. 
Table 3 Genomic map and characteristics of putative MuLV-ERVs harboring the unique U3 sequences

\begin{tabular}{|c|c|c|c|c|c|c|c|c|c|c|c|c|}
\hline \multirow{2}{*}{ No } & \multirow{2}{*}{ Virus } & \multirow{2}{*}{ Chr } & \multicolumn{2}{|c|}{ Location } & \multirow{2}{*}{ Ori } & \multirow{2}{*}{$\begin{array}{c}\text { Virus } \\
\text { size (bp) }\end{array}$} & \multirow{2}{*}{ PBS } & \multicolumn{3}{|c|}{ ORF } & \multirow{2}{*}{$\begin{array}{l}\text { Occurrence } \\
\text { frequency }\end{array}$} & \multirow{2}{*}{ Reference } \\
\hline & & & From & To & & & & gag & pol & env & & \\
\hline 1 & MuERV-49 & 1 & 133539144 & 133548197 & + & 9054 & Q & - & + & + & $U / U$ & $\mathrm{I}, \mathrm{Il}, \mathrm{II}, \mathrm{IV}$ \\
\hline 2 & MuERV-31 & 1 & 166153938 & 166159683 & + & 5746 & Q & + & - & - & $\mathrm{U} / \mathrm{T}$ & I,II,IV \\
\hline 3 & MuERV-30 & 1 & 184187251 & 184196231 & + & 8981 & Q & + & $P$ & + & $\mathrm{U} / \mathrm{T}$ & $\mathrm{I}, \mathrm{Il}, \mathrm{IV}$ \\
\hline 4 & MuERV-33 & 1 & 193757492 & 193766472 & + & 8981 & Q & + & $P$ & - & $\mathrm{U} / \mathrm{T}$ & I,II,IV \\
\hline 5 & MuERV-35 & 2 & 57066823 & 57075803 & - & 8981 & Q & + & + & + & $\mathrm{U} / \mathrm{T}$ & I,II,IV \\
\hline 6 & MuERV-3 & 2 & 156182292 & 156189341 & - & 7050 & Q & + & - & + & $\mathrm{U} / \mathrm{U}$ & \|\|$, I V$ \\
\hline 7 & MuERV-48 & 3 & 66900000 & 66907716 & + & 7717 & Q & + & - & - & $U / U$ & $\mathrm{I}, \mathrm{Il}, \mathrm{II}, \mathrm{IV}$ \\
\hline 8 & MuERV-55 & 3 & 151986104 & 151995146 & + & 9043 & Q & + & + & + & $U / U$ & $\mathrm{I}, \mathrm{Il}, \mathrm{II}, \mathrm{IV}$ \\
\hline 9 & MuERV-29 & 4 & 101535762 & 101544743 & - & 8982 & Q & + & $P$ & + & $\mathrm{U} / \mathrm{T}$ & $\mathrm{I}, \|, \mathrm{IV}$ \\
\hline 10 & MuERV-28 & 4 & 107818639 & 107827621 & - & 8983 & Q & + & + & + & $\mathrm{U} / \mathrm{T}$ & I,II,IV \\
\hline 11 & MuERV-2 & 4 & 132651872 & 132657538 & + & 5667 & Q & - & - & - & $U / U$ & $\mathrm{I}, \mathrm{Il}, \mathrm{II}, \mathrm{IV}$ \\
\hline 12 & MuERV-6 & 4 & 133715306 & 133720617 & + & 5312 & Q & + & - & - & $\mathrm{U} / \mathrm{U}$ & $\mathrm{I}, \mathrm{I}, \mathrm{II} \mid \mathrm{I}, \mathrm{IV}$ \\
\hline 13 & MuLV_ERV ${ }_{4-5}{ }^{*}$ & 4 & 145686198 & 145694956 & - & 8759 & Q & + & + & + & $U / U$ & this study \\
\hline 14 & XMV9 & 4 & 146592937 & 146601658 & + & 8722 & Q & + & + & + & $\mathrm{U} / \mathrm{U}$ & V \\
\hline 15 & $L-3-9.5$ & 5 & 23206397 & 23215063 & - & 8667 & $P$ & + & + & + & $\mathrm{U} / \mathrm{U}$ & I,II \\
\hline 16 & MuLV_ERV ${ }_{5-2^{*}}$ & 5 & 23721126 & 23727971 & - & 6846 & Q & + & - & + & $U / U$ & this study \\
\hline 17 & MuERV-27 & 5 & 33864636 & 33873616 & + & 8981 & Q & - & + & + & $\mathrm{U} / \mathrm{T}$ & $\mathrm{I}, \mathrm{II}, \mathrm{IV}$ \\
\hline 18 & MuERV-18 & 5 & 44525599 & 44534580 & + & 8982 & Q & - & + & + & $\mathrm{U} / \mathrm{T}$ & $\mathrm{I}, \mathrm{I}, \mathrm{IV}$ \\
\hline 19 & MuERV-19 & 5 & 77359541 & 77368522 & + & 8982 & Q & + & + & + & $\mathrm{U} / \mathrm{T}$ & I,IIIIV \\
\hline 20 & MuERV-26 & 7 & 7022622 & 7031603 & - & 8982 & Q & $P$ & - & - & $\mathrm{U} / \mathrm{T}$ & I,II,IV \\
\hline 21 & MuERV-24 & 7 & 30400226 & 30408924 & + & 8699 & Q & + & + & $P$ & $\mathrm{U} / \mathrm{T}$ & $\mathrm{I}, \mathrm{I}, \mathrm{IV}$ \\
\hline 22 & MuERV-25 & 7 & 31473659 & 31482639 & + & 8981 & Q & + & $P$ & - & $\mathrm{U} / \mathrm{T}$ & I,II,IV \\
\hline 23 & MuERV-34 & 7 & 123575623 & 123581296 & + & 5674 & Q & + & - & - & $\mathrm{U} / \mathrm{T}$ & $\mathrm{I}, \mathrm{I}, \mathrm{IV}$ \\
\hline 24 & MuERV-10 & 8 & 44397084 & 44405817 & - & 8734 & Q & + & + & + & $\mathrm{U} / \mathrm{T}$ & I,IIIIV \\
\hline 25 & MuERV-11 & 8 & 87649754 & 87656141 & - & 6388 & Q & + & $P$ & - & $\mathrm{U} / \mathrm{T}$ & I,II,IV \\
\hline 26 & MuERV-1 & 8 & 93410190 & 93415857 & + & 5668 & Q & - & - & - & $\mathrm{U} / \mathrm{U}$ & $\mathrm{I}, \mathrm{Il}, \mathrm{II}, \mathrm{IV}$ \\
\hline 27 & MuERV-12 & 8 & 122413465 & 122420940 & - & 7476 & Q & - & - & + & $\mathrm{U} / \mathrm{T}$ & $\mathrm{I}, \mathrm{Il}, \mathrm{IV}$ \\
\hline 28 & MuERV-8 & 9 & 41713568 & 41720351 & - & 6784 & Q & - & + & + & $U / U$ & $\mathrm{I}, \mathrm{Il}, \mathrm{IV}$ \\
\hline 29 & iLN(III)-1a & 9 & 62286981 & 62295739 & + & 8759 & Q & + & + & + & $U / U$ & 1 \\
\hline 30 & K-4-11.10a & 10 & 8263933 & 8271066 & - & 7134 & Q & - & - & - & $\mathrm{U} / \mathrm{T}$ & | II \\
\hline 31 & MuERV-53 & 10 & 22454084 & 22463128 & + & 9045 & Q & + & + & + & $\mathrm{U} / \mathrm{U}$ & $\mathrm{I}, \mathrm{Il}, \mathrm{II}, \mathrm{IV}$ \\
\hline 32 & MuERV-17 & 10 & 41156454 & 41165434 & + & 8981 & Q & + & + & + & $\mathrm{U} / \mathrm{T}$ & $\mathrm{I}, \mathrm{I}, \mathrm{IV}$ \\
\hline 33 & BM-a-2.11e & 11 & 5905655 & 5911518 & + & 5864 & Q & - & - & - & $\mathrm{B} / \mathrm{B}$ & III \\
\hline 34 & MuERV-22 & 11 & 6649425 & 6658394 & - & 8970 & Q & + & - & + & $\mathrm{U} / \mathrm{T}$ & I,IIIIV \\
\hline 35 & MuERV-23 & 11 & 820301 & 8829280 & + & 8980 & Q & + & + & + & $\mathrm{U} / \mathrm{T}$ & I,II,IV \\
\hline 36 & MuERV-50 & 11 & 60392151 & 60399510 & - & 7360 & Q & - & $P$ & - & $U / U$ & $\mathrm{I}, \mathrm{Il}, \mathrm{II}, \mathrm{IV}$ \\
\hline 37 & MuERV-51 & 11 & 76362310 & 76371354 & + & 9045 & Q & + & + & + & $U / U$ & $\mathrm{I}, \mathrm{II}, \mathrm{II}, \mathrm{IV}$ \\
\hline 38 & MuERV-38 & 11 & 88751494 & 88758280 & - & 6787 & Q & + & - & + & $U / T$ & $\mathrm{I}, \|, \mathrm{IV}$ \\
\hline 39 & MuERV-37 & 12 & 20704753 & 20710513 & + & 5761 & Q & - & - & - & $\mathrm{U} / \mathrm{T}$ & I,II,IV \\
\hline 40 & MuERV-16 & 12 & 55875211 & 55884192 & + & 8982 & Q & + & + & + & $\mathrm{U} / \mathrm{T}$ & IV \\
\hline 41 & MuERV-5 & 13 & 67985825 & 67994511 & - & 8687 & Q & + & + & + & $\mathrm{U} / \mathrm{U}$ & $\mathrm{I}, \mathrm{Il}, \mathrm{II}, \mathrm{IV}$ \\
\hline 42 & MuERV-21 & 13 & 99756230 & 99765210 & - & 8981 & Q & + & $P$ & + & $\mathrm{U} / \mathrm{T}$ & $\mathrm{I}, \mathrm{I}, \mathrm{IV}$ \\
\hline 43 & MuERV-7 & 14 & 54912048 & 54917591 & - & 5544 & Q & + & - & - & $U / U$ & IV \\
\hline 44 & $L-1-2.14$ & 14 & 55149203 & 55157025 & - & 7823 & Q & $P$ & + & + & $U / U$ & I,II \\
\hline 45 & MuERV-15 & 15 & 76390247 & 76397432 & - & 7186 & Q & - & - & + & $\mathrm{U} / \mathrm{T}$ & I,II,IV \\
\hline 46 & U-1-5.16 & 16 & 76135288 & 76144269 & - & 8982 & Q & - & $P$ & - & $\mathrm{U} / \mathrm{T}$ & I,II \\
\hline
\end{tabular}


Table 3 Genomic map and characteristics of putative MuLV-ERVs harboring the unique U3 sequences (Continued)

\begin{tabular}{|c|c|c|c|c|c|c|c|c|c|c|c|c|}
\hline 47 & MuERV-14 & 16 & 93697702 & 93706682 & - & 8981 & Q & - & + & + & $\mathrm{U} / \mathrm{T}$ & $\mathrm{I}, \mathrm{IV}$ \\
\hline 48 & MuERV-20 & 18 & 82862943 & 82871822 & + & 8880 & $Q$ & - & + & + & $\mathrm{U} / \mathrm{T}$ & I,IIIIV \\
\hline 49 & MuERV-32 & 19 & 38449512 & 38456573 & + & 7062 & Q & - & $P$ & - & $\mathrm{U} / \mathrm{T}$ & I,IIIIV \\
\hline 50 & MuERV-9 & 19 & 61001210 & 61009936 & - & 8727 & $Q$ & + & + & + & $\mathrm{U} / \mathrm{U}$ & $\mathrm{I}, \|, \mathrm{IIV}$ \\
\hline 51 & MuERV-13 & $x$ & 15051174 & 15060154 & + & 8981 & Q & + & $P$ & + & $U / T$ & $\mathrm{I}, \mathrm{II}, \mathrm{IV}$ \\
\hline
\end{tabular}

Chr (chromosome), Ori (orientation), PBS (primer binding site), Q (tRNA $\left.{ }^{\text {glutamine }}\right), \mathrm{P}$ (tRNA ${ }^{\text {proline }}$ ), ORF (open reading frame), + (intact/full-length), - (defective), U (both B- and T- cells; both no burn and burn), B (B-cell), T (T-cell), references I [14], II [33], III [31], IV [30] and V [32]. *MuLV-ERV newly identified in this study. The names of MuLV-ERVs, which have been reported in previous studies, are adapted from the relevant reference in bold.

was confirmed by its negative score for the Tajima's Neutrality test in comparison to the positive values of the other two groups [24]. The results from the second diversity analysis demonstrated that there is a decrease in U3 population diversity in both burn-24 hours-B-cells and burn-24 hours-T-cells groups in comparison to the no burn controls. Furthermore, it was apparent that at the 24 hours time point, the U3 population in B-cells from both no burn and burn were less diverse in comparison to T-cells' U3 population.

The findings from these diversity analyses indicate that burn-elicited stressors lead to the induction of specific MuLV-ERVs in a time after injury-dependent manner, and the population of MuLV-ERVs expressed in T-cells is more heterogeneous than the population in B-cells.

\section{Differential transcription potentials of MuLV-ERV U3-promoter sequences}

The U3 sequence on the $5^{\prime}$ LTR, which is presumed to be identical to the U3 of the $3^{\prime}$ LTR, serves as an ERV promoter. In this study, the transcription potentials of the 100 unique U3-promoter sequences were surveyed for transcription regulatory elements (TREs). Nine TREs were predominantly present in the $\mathrm{U} 3$ sequences isolated from both B- and T-cells of burn mice at the 3 hours and/ or 24 hours time points compared to the U3 sequences from no burn mice (Table 2 and Additional file 2: Table S2). Among the nine TREs are BRNF (Brn POU domain factors), AIRE (Autoimmune regulatory element binding factor), and NRF1 (Nuclear respiratory factor 1), which have been reported to be linked to various stress responses $[25,26]$. Furthermore, it appears that three TREs occurred more often in the U3 sequences of B-cells than T-cells whereas the U3 sequences from T-cells had a higher frequency of 16 other TREs in comparison to B-cells (Table 2 and Additional file 2: Table S2). Among the 16 TREs, MAZF (Myc associated zinc fingers), GATA (GATA binding factors) and MEF2 (myocyte-specific enhancer binding factor) have been reported to be involved in T-cell activation [27-29]. A total of 122 TREs, including the 28 TREs described above, were identified from the 100 unique sequences, and the comprehensive TRE profiles were summarized in Additional file 2: Table S2 and Additional file 3: Table S3.
It is probable that the TREs (e.g., BRNF, AIRE, NRF1), which were mapped on the U3 sequences almost exclusively derived from burn mice, are closely associated with the modulation of MuLV-ERV expression in conjunction with a transcription environment dynamically formulated by burn-elicited stressors.

\section{In silico cloning and characterization of putative MuLV-ERVs harboring the unique U3 sequences}

To investigate the biological properties of the putative MuLV-ERVs harboring the U3 sequences identified in this study, the C57BL/6J mouse genome was surveyed to map the proviral loci using the 100 unique U3 sequences as mining probes. From the in silico mining experiment, 49 ERVs were found with two identical LTR sequences at both ends and two, MuERV-49 and MuERV-26, retained slightly different LTR sequences due to a few point mutations. Forty-nine of the 51 putative MuLVERVs identified from this survey, including 15 full-length proviruses retaining a set of intact coding potentials for gag, pol and env polypeptides, have been reported in previous studies [14,30-33]. The two newly identified MuLV-ERVs are: one full-length provirus (8,759 nucleotides) with intact coding potentials and one defective provirus (6,846 nucleotides) (Table 3). An analysis of the primer binding site revealed that both MuLV-ERVs had the $\mathrm{tRNA}^{\mathrm{Gln}}$ priming sequence. All 51 putative MuLVERVs were mapped using the U3 probes derived from both no burn and burn mice, except for BM-a-2.11e [31], which was identified using a U3 probe unique for B-cells of burn mice. On the other hand, 30 putative MuLV-ERV loci were retrieved using U3 probes exclusively isolated from T-cells. Future studies focusing on the biological properties of these putative MuLV-ERVs would be of interest: 1) effects (e.g., cytotoxicity) of their replication on immune cells in conjunction with infection followed by random integration into the genome and 2) functions (e.g., regulation of inflammatory mediators) of their gene products ( $\mathrm{gag}$, pol, and env polypeptides).

\section{Identification of genes neighboring the MuLV-ERV loci}

The U3 promoter and the enhancer sequences embedded in MuLV-ERVs can affect the transcriptional activities of 
the neighboring genes via the promotion/repression of transcription, and alternative splicing and polyadenylation [34]. In this study, we identified the genes located within $100 \mathrm{~Kb}$ upstream and downstream of each MuLV-ERV integration site. Of a total of 131 genes identified, nine genes had MuLV-ERVs integrated into their introns (Additional file 4: Table S4). It will be interesting to examine how the expression patterns (depending on lymphocyte type or burn-elicited stress) of these proviruses are correlated with the expression of the relevant genes, for example, the relationship between BM-a-2.11e MuLV-ERV, which was identified using a U3 probe uniquely identified from the B-cells of burn mice, and Camk2b (calcium/calmodulindependent protein kinase II-beta) locus. The Camk2b expression is associated with T-cell activation and cytotoxic T-cell activity $[35,36]$. It is possible that the burn-elicited stressors may affect the promoter and/or enhancer activities of certain MuLV-ERVs paralleling modulation of the expression of adjacent genes in a specific lymphocyte type.

\section{Conclusion}

It is expected that burn injury elicits a range of stress signals that are directly linked to pathologic changes in the immune system and other organs [37]. The majority of the underlying mechanisms regarding how these stressors contribute to post-burn pathogenesis still remain to be characterized. The findings from this study suggest that certain MuLV-ERVs are differentially modulated depending on the lymphocyte type, location of lymphoid organ, and duration after injury in conjunction with the burnelicited stressors. As a result of activation of certain MuLV-ERVs following burn injury, the gene products and/or newly produced virus particles of the activated ERVs may participate in specific burn pathogeneses, such as immune disorder and cytotoxicity, in a cell type-specific manner. In fact, our laboratory has reported that some MuLV-ERVs are activated in the liver of mice after burn injury which resulted in evident hepatic damage [33]. Further investigations are needed to elucidate the link between lymphocyte type-specific post-burn MuLV-ERV activation and systemic inflammatory disorder as well as the other aspects of burn pathogeneses.

\section{Additional files}

Additional file 1: Table S1. Sources of the 100 MuLV-ERV U3 sequences analyzed in this study. Yellow and gray highlights indicate unique MuLV-ERV U3 sequences and clones without sequence information, respectively. MAN (mandibular cervical LN), ACC (accessory mandibular cervical LN), SUP (superficial parotid cervical LN), DEE (deep cervical LN) LN (lymph node), SM (size marker).

Additional file 2: Table S2. The profile of transcription regulatory elements in 100 unique MuLV-ERV U3-promoter sequences. The number in the box indicates the occurrence frequency of each transcription regulatory element (TRE). Colored boxes indicate TREs predominantly identified in the U3 sequences derived from specific experimental groups: burn group (green), B-cell group (yellow), and T-cell group (blue).

Additional file 3: Table S3. Abbreviations: transcription regulatory elements.

Additional file 4: Table S4. Annotated genes neighboring $100 \mathrm{~Kb}$ upstream and downstream of the individual MuLV-ERV integration loci. Chr (Chromosome number), Ori (Orientation). Gray highlight indicates the annotated genes harboring an ERV in the intron. *MuLV-ERV newly identified in this study. Please refer to Table 3 for the MuLV-ERV names.

\section{Abbreviations}

HERV: Human endogenous retroviruses; LN: Lymph node; LTR: Long terminal repeat; MuLV-ERVs: Murine leukemia virus-type endogenous retroviruses; PBS: Primer binding site; TREs: Transcription regulatory elements.

\section{Competing interests}

There is no conflicting financial interest.

\section{Authors' contributions}

This study was conceived and managed by KC. DGG participated in scientific discussions. $\mathrm{KHL}, \mathrm{DL}$, and TG performed the experiments. $\mathrm{KHL}$ generated the figures and drafted the manuscript. All authors read and approved the final manuscript.

\section{Acknowledgement}

This study was supported by grants from Shriners of North America (No. 86800 to KC and No. 84302 to KHL [postdoctoral fellowship]) and the National Institutes of Health (R01 GM071360 to KC).

Received: 19 April 2012 Accepted: 11 December 2012

Published: 5 January 2013

\section{References}

1. Waterston RH, Lindblad-Toh K, Birney E, Rogers J, Abril JF, Agarwal P, Agarwala R, Ainscough R, Alexandersson M, An P, et al: Initial sequencing and comparative analysis of the mouse genome. Nature 2002, 420:520-562.

2. Mayer J, Sauter M, Racz A, Scherer D, Mueller-Lantzsch N, Meese E: An almost-intact human endogenous retrovirus $\mathrm{K}$ on human chromosome 7. Nat Genet 1999, 21:257-258.

3. Mueller-Lantzsch N, Sauter M, Weiskircher A, Kramer K, Best B, Buck M, Grasser F: Human endogenous retroviral element K 10 (HERV-K 10) encodes a full-length gag homologous $73-\mathrm{kDa}$ protein and a functional protease. AIDS Res Hum Retroviruses 1993, 9:343-350.

4. Vogt VM: Retroviral Virions and Genomes. In Retroviruses. Edited by Coffin JM, Hughes SH, Varmus HE. Cold Spring Harbor (NY): Cold Spring Harbor Laboratory Press; 1997:27-69.

5. Lavie L, Kitova M, Maldener E, Meese E, Mayer J: CpG methylation directly regulates transcriptional activity of the human endogenous retrovirus family HERV-K (HML-2). J Virol 2005, 79:876-883.

6. Moyes DL, Martin A, Sawcer S, Temperton N, Worthington J, Griffiths DJ, Venables PJ: The distribution of the endogenous retroviruses HERV-K113 and HERV-K115 in health and disease. Genomics 2005, 86:337-341.

7. Frendo JL, Olivier D, Cheynet V, Blond JL, Bouton O, Vidaud M, Rabreau M, Evain-Brion D, Mallet F: Direct involvement of HERV-W Env glycoprotein in human trophoblast cell fusion and differentiation. Mol Cell Biol 2003, 23:3566-3574.

8. Mameli G, Astone V, Arru G, Marconi S, Lovato L, Serra C, Sotgiu S, Bonetti B, Dolei A: Brains and peripheral blood mononuclear cells of multiple sclerosis (MS) patients hyperexpress MS-associated retrovirus/HERV-W endogenous retrovirus, but not Human herpesvirus 6. J Gen Virol 2007, 88:264.

9. Purcell EM, Dolan SM, Kriynovich S, Mannick JA, Lederer JA: Burn injury induces an early activation response by lymph node CD4+ T-cells. Shock 2006, 25:135.

10. Schneider DF, Glenn CH, Faunce DE: Innate lymphocyte subsets and their immunoregulatory roles in burn injury and sepsis. J Burn Care Res 2007, 28:365-379.

11. Maldonado MD, Venturoli A, Franco A, Nunez-Roldan A: Specific changes in peripheral blood lymphocyte phenotype from burn patients. Probable 
origin of the thermal injury-related lymphocytopenia. Burns 1991, 17:188-192

12. Barlow $Y: T$ lymphocytes and immunosuppression in the burned patient: a review. Burns 1994, 20:487-490.

13. Hotchkiss RS, Tinsley KW, Swanson PE, Schmieg RE, Hui JJ, Chang KC, Osborne DF, Freeman BD, Cobb JP, Buchman TG: Sepsis-induced apoptosis causes progressive profound depletion of $B$ and CD4+ T lymphocytes in humans 1. J Immunol 2001, 166:6952-6963.

14. Lee YK, Chew A, Phan H, Greenhalgh DG, Cho K: Genome-wide expression profiles of endogenous retroviruses in lymphoid tissues and their biological properties. Virology 2008, 373:263-273.

15. Lee YK, Chew A, Greenhalgh DG, Cho K: Tropism, cytotoxicity, and inflammatory properties of two envelope genes of murine leukemia virus type-endogenous retroviruses of C57BL/6J mice. Mediators Inflamm 2011, 2011:509604

16. Cho K, Adamson LK, Greenhalgh DG: Induction of murine AIDS virusrelated sequences after burn injury. J Surg Res 2002, 104:53-62.

17. Tomonaga $\mathrm{K}$, Coffin JM: Structures of endogenous nonecotropic murine leukemia virus (MLV) long terminal repeats in wild mice: implication for evolution of MLVs. J Virol 1999, 73:4327-4340.

18. Bingham J, Sudarsanam S: Visualizing large hierarchical clusters in hyperbolic space. Bioinformatics 2000, 16:660-661.

19. Tomonaga K, Coffin JM: Structure and distribution of endogenous nonecotropic murine leukemia viruses in wild mice. J Virol 1998, 72:8289-8300.

20. Quandt K, Frech K, Karas H, Wingender E, Werner T: MatInd and MatInspector: new fast and versatile tools for detection of consensus matches in nucleotide sequence data. Nucleic Acids Res 1995, 23:4878-4884.

21. Tamura K, Dudley J, Nei M, Kumar S: MEGA4: molecular evolutionary genetics analysis (MEGA) software version 4.0. Mol Biol Evol 2007, 24:1596-1599.

22. Nikbakht KN, Ou CY, Boone LR, Glover PL, Yang WK: Nucleotide sequence analysis of endogenous murine leukemia virus-related proviral clones reveals primer-binding sites for glutamine tRNA. J Virol 1985, 54:889-893.

23. Kotadia BK, Ravindranath TM, Choudhry MA, Haque F, Al-Ghoul W, Sayeed MM: Effects of pentoxyfylline on mesenteric lymph node T-cells in a rat model of thermal injury. Shock 2003, 20:517-520.

24. Tajima F: DNA polymorphism in a subdivided population: the expected number of segregating sites in the two-subpopulation model. Genetics 1989, 123:229-240.

25. Kovacs KJ, Sawchenko PE: Sequence of stress-induced alterations in indices of synaptic and transcriptional activation in parvocellular neurosecretory neurons. J Neurosci 1996, 16:262-273.

26. Suliman HB, Carraway MS, Welty-Wolf KE, Whorton AR, Piantadosi CA: Lipopolysaccharide stimulates mitochondrial biogenesis via activation of nuclear respiratory factor-1. J Biol Chem 2003, 278:41510-41518.

27. Duncan DD, Stupakoff A, Hedrick SM, Marcu KB, Siu G: A Myc-associated zinc finger protein binding site is one of four important functional regions in the CD4 promoter. Mol Cell Biol 1995, 15:3179-3186.

28. Ting CN, Olson MC, Barton KP, Leiden JM: Transcription factor GATA-3 is required for development of the T-cell lineage. Nature 1996, 384:474-478.

29. Volker JL, Rameh LE, Zhu Q, DeCaprio J, Hansen U: Mitogenic stimulation of resting T-cells causes rapid phosphorylation of the transcription factor LSF and increased DNA-binding activity. Genes Dev 1997, 11:1435-1446.

30. Kwon DN, Lee YK, Greenhalgh DG, Cho K: Lipopolysaccharide stress induces cell-type specific production of murine leukemia virus typeendogenous retroviral virions in primary lymphoid cells. J Gen Virol 2011, 92:292-300

31. Kwon DN, Greenhalgh DG, Cho K: Cloning and characterization of endogenous retroviruses associated with postinjury stress signals in lymphoid tissues. Shock 2009, 32:80.

32. Stoye JP, Kaushik N, Jeremiah S, Best S: Genetic map of the region surrounding the retrovirus restriction locus, Fv1, on mouse chromosome 4. Mamm Genome 1995, 6:31-36.

33. Lee YK, Chew A, Fitzsimon L, Thomas R, Greenhalgh D, Cho K: Genomewide changes in expression profile of murine endogenous retroviruses (MuERVs) in distant organs after burn injury. BMC Genomics 2007, 8:440.

34. Selten G, Cuypers HT, Berns A: Proviral activation of the putative oncogene Pim-1 in MuLV induced T-cell lymphomas. EMBO J 1985, 4:1793.
35. Mack KD, Von Goetz M, Lin M, Venegas M, Barnhart J, Lu Y, Lamar B, Stull R, Silvin C, Owings $P$, et al: Functional identification of kinases essential for T-cell activation through a genetic suppression screen. Immunol Lett 2005, 96:129-145.

36. Liu X, Yao M, Li N, Wang C, Zheng Y, Cao X: CaMKIl promotes TLRtriggered proinflammatory cytokine and type I interferon production by directly binding and activating TAK1 and IRF3 in macrophages. Blood 2008, 112:4961-4970.

37. Deitch EA: Role of the gut lymphatic system in multiple organ failure. Curr Opin Crit Care 2001, 7:92.

doi:10.1186/1471-2172-14-2

Cite this article as: Lee et al: Injury-elicited stressors alter endogenous retrovirus expression in lymphocytes depending on cell type and source lymphoid organ. BMC Immunology 2013 14:2.

\section{Submit your next manuscript to BioMed Central and take full advantage of:}

- Convenient online submission

- Thorough peer review

- No space constraints or color figure charges

- Immediate publication on acceptance

- Inclusion in PubMed, CAS, Scopus and Google Scholar

- Research which is freely available for redistribution
C Biomed Central 